\title{
Biochemically Recurrent Prostate
} Carcinoma

National Cancer Institute

\section{Source}

National Cancer Institute. Biochemically Recurrent Prostate Carcinoma. NCI Thesaurus.

Code C156771.

Increasing prostate-specific antigen (PSA) following prostatectomy or radiation therapy in a patient with a history of localized prostate carcinoma. Signs of metastasis are absent using the currently available scanning technology. 